respectively. Hypoparathyroidism's etiologies were: autoimmune polyendocrine syndrome (2cases), idiopathic hypoparathyroidism (3cases), kearns sayer syndrome (1cas). PHP was diagnosed in 3 cases; among them, 2 children had Fahr syndrome. All patients were treated with oral calcium, active vitamin D. 2 patients died; the cause of death was not related to their HP.

Conclusion HP is a rare endocrinopathy in childhood. The etiological diagnosis strategy needs many investigations especially genetic analysis.

\section{BONE MINERAL DENSITY IN CHILDREN WITH CEREBRAL PALSY}

doi:10.1136/archdischild-2012-302724.0660

${ }^{1} \mathrm{~V}$ Toopchizadeh, ${ }^{2} \mathrm{M}$ Barzegar. 'Physical Medicine \& Rehabilitation Research Center, Tabriz University of Medical Sciences, ${ }^{2}$ Pediatrics, Tabriz University of Medical Sciences, Pediatric Health Research Center, Tabriz, Iran

Diminished bone mineral density (BMD) is a frequent finding in cerebral palsied children. The underlying pathophysiology is complex in CP and variable risk factors such as immobility, nutritional deficiency and anticonvulsant use have been proposed. This study aimed at assessing BMD in children with CP.

In this analytic-descriptive cross-sectional study, 85 children with CP were recruited in Tabriz Children Teaching Centre. Patients' $\mathrm{BMD}$ of three lumbar vertebrae (L2-L4) and hip was determined by Dual energy X-ray Absorptiometry (DXA). Functional status was assessed by the Gross Motor Functional Classification (GMFC) scale. Eighty five patients, 44 males and 41 females with a mean age of $5.79 \pm 2.39$ (3-11) years were enrolled in the study. Reduced BMD, $-2<Z$ score $<-1$ and $Z$ score $\leq-2$ were present in $48.2 \%$ and $30.6 \%$ of the patients, respectively. Standing ability and its duration was directly associated with increased lumbar vertebral BMD. The mean hip BMD was significantly lower in the cases with positive history of receiving anti-convulsant medications. The mean lumbar vertebral and hip BMDs were higher in cases with hypotonic CP and in the patients with hemiplegic involvement in a nonsignificant manner. There was also nonsignificant inverse correlation between the $\mathrm{BMD}$ and GMFC.

Diminished $\mathrm{BMD},-2<\mathrm{Z}$ score $<-1$ and $\mathrm{Z}$ score $\leq-2$ are frequent in children with CP. The relating pathophysiology is multifactorial and complex.

\section{VITAMIN A AND IRON AS AN ADJUVANT THERAPY IN ADDITION OF GNRH AGONIST IN PRECOCIOUS PUBERTY}

doi:10.1136/archdischild-2012-302724.0661

'Z Karamizadeh, ${ }^{2} \mathrm{H}$ Mohammadi. 'Endocrinology; ${ }^{2}$ Shiraz University of Medical Sciences, Shiraz, Iran

Background To examine the effect of vitamin A and Iron supplementation on growth outcome of central precocious puberty (CPP) patients who receive $\mathrm{GnRH}$ agonist.

Method 36 female CPP patients were randomized in control (17 cases) and trial (19 cases) groups. Both groups received $\mathrm{GnRH}$ agonist and the trial group received iron (10m/day) and vitamin A 6000 $\mathrm{U} /$ week as well. The patients were revisited every 3 months, their weights, height, BMI were measured, and their bone age was determined in the beginning and end of the study. Statistical analysis was performed between groups and in each group.

Results The mean age of the patients was $106.7 \pm 10.57$ vs. 102.7 \pm 13.7 months in trial and control groups. No statistical difference was observed in the base-line age, weight, height, BMI, bone age and predicted adult height (PAH). Height $Z$ score (1.22 \pm 0.9 vs. $0.39 \pm 0.7$, p value $<0.01)$, and height velocity $Z$ score $(1.4 \pm 2.1 \mathrm{vs}$ $-0.36 \pm 1.9$, $p$ value $<0.01)$ were significantly higher in the trial as compared to the control group at the end of the study. PAH-SDS had no significant changes in each group and between the two groups (trial: $-0.29 \pm 0.9$ vs. control: $-0.95 \pm 1.1$, p value $>0.05$ ). Height Velocity with age had a negative linear correlation and height $Z$ score was positively related to the initial height and weight ( $p$ value $<0.05$ ) in both groups.

Conclusion In CPP, adjuvant therapy with Vitamin A and iron in combination with $\mathrm{GnRH}$ agonist could be considered to improve height velocity.

\section{IMAGE SYNDROME: FIRST NORTH AFRICAN DESCRIPTION}

doi:10.1136/archdischild-2012-302724.0662

${ }^{1} \mathrm{~N}$ Boutrid, ${ }^{1} \mathrm{H}$ Rahmoune, ${ }^{2} \mathrm{H}$ Boutrid, ${ }^{3} \mathrm{~N}$ Boughaba, ${ }^{4} \mathrm{FC}$ Rahmoune, ${ }^{1} \mathrm{~B}$ Bioud. ${ }^{1}$ Pediatrics, University Hospital of Setif, Setif; ${ }^{2}$ Obstetrics \& Gynecology, Bab El-Oued University Hospital, Algiers; ${ }^{3}$ Pediatric Surgery, EHS Mère-Enfant, Constantine, Algeria; ${ }^{4}$ Anesthésie-Réanimation, AP-HP, Hôpital Bicêtre, Paris, France

Introduction If the diagnosis of adrenal insufficiency (IS) with salt loss is quite common, some forms deserve particular attention. Description A 40 days infant, twin born SGA is admitted for severe dehydration with impaired general condition. The physical examination found a right inguinal hernia and hypospadias. No dysmorphia is highlighted.

The assessment carried outhypoglycemia with hyponatremia and hyperkalemia. Rehydration with IV corticosteroid therapy is then initiated based hydrocrtisone.

The association Inguinal Hernia-IS-Hypospadias is a rare form of congenital deficiencies in adrenal enzymes: IMAGe syndrome associating intrauterine growth retardation (I), metaphyseal dysplasia $(\mathrm{M})$, adrenal hypoplasia (A), genital abnormalities (Ge).

A radiograph of the members is requested and returns, at first, normal; but progressive dysplasia is noted after 1 year.

Discussion The causes of neonatal adrenal insufficiency are a heterogenous group.

The sexual ambiguity, the association with clinical and laboratory diagnosis led to a such rare diagnosis. Genetic analysis was not yet performed.

The acronym IMAGe (OMIM 300290) was individualized in 1999 by Naughty \& Al.

This condition can be added later by a dyplasie metaphyseal, but the exact cause remains unknown In 2008, a Japanese team emphasized the radiological monitoring of these children.

Conclusion Advances in genetics still perplexing in some forms of neonatal IS, despite the role better and better established SF-1 gene DAX-1 \& IMAGe syndrome is a perfect example: association of clinical and radiological signs of unknown origin described in the five continents.

\section{FIRST SEIZURE AS LATE PRESENTATION OF VELO-CARDIO- FACIAL SYNDROME}

doi:10.1136/archdischild-2012-302724.0663

II Simão, ${ }^{2} \mathrm{~T}$ Lourenço, ${ }^{3} \mathrm{~L}$ Lopes, ${ }^{4} \mathrm{MP}$ Ramos. ${ }^{1}$ Centro Hospitalar Lisboa Central - Hospital

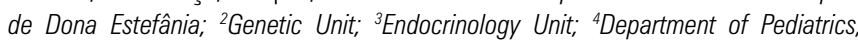
H. Dona Estefânia, Centro Hospitalar Lisboa Central, Lisbon, Portugal

Congenital hypoparathyroidism caused by Velo-Cardio-Facial Syndrome (VCFS) typically presents with hypocalcemia usually associated with other characteristic findings. It is most often diagnosed during the neonatal period.

We report the case of a boy who presented with symptomatic hypocalcemia (serum total calcium $5.1 \mathrm{mg} / \mathrm{dL}$; ionized calcium $0.65 \mathrm{mmol} / \mathrm{L}$ ) at 8 years of age; he had a history of velopharyngeal incompetence and late development with language impairment; at presentation, mild dysmorphic facial features were detected. The laboratory evaluation revealed parathormone $(\mathrm{PTH})<1.0 \mathrm{pg} / \mathrm{ml}$. The presence of hypoparathyroidism suggested VCFS, confirmed by 